\title{
Co-expressed microRNAs, target genes and pathways related to metabolism, inflammation and endocrine function in individuals at risk for type 2 diabetes
}

\author{
ELENA FLOWERS ${ }^{1,2}$, KESAVA ASAM $^{3}$, ISABEL ELAINE ALLEN ${ }^{4}$, \\ ALKA M. KANAYA ${ }^{4,5}$ and BRADLEY E. AOUIZERAT ${ }^{3,6}$
}

\begin{abstract}
${ }^{1}$ Department of Physiological Nursing; ${ }^{2}$ Institute for Human Genetics, University of California San Francisco, San Francisco, CA 94143-0610; ${ }^{3}$ Bluestone Center for Clinical Research, New York University, New York, NY 10010; Departments of ${ }^{4}$ Epidemiology and Biostatistics and ${ }^{5}$ Medicine, University of California San Francisco, San Francisco, CA 94143-0610; ${ }^{6}$ Department of Oral and Maxillofacial Surgery, New York University, New York, NY 10010, USA
\end{abstract}

Received November 5, 2021; Accepted February 3, 2022

DOI: $10.3892 / \mathrm{mmr} .2022 .12672$

\begin{abstract}
MicroRNAs (miRNAs) may be considered important regulators of risk for type 2 diabetes (T2D). The aim of the present study was to identify novel sets of miRNAs associated with T2D risk, as well as their gene and pathway targets. Circulating miRNAs $(n=59)$ were measured in plasma from participants in a previously completed clinical trial $(n=82)$. An agnostic statistical approach was applied to identify novel sets of miRNAs with optimal co-expression patterns. In silico analyses were used to identify the messenger RNA and biological pathway targets of the miRNAs within each factor. A total of three factors of miRNAs were identified, containing 18, seven and two miRNAs each. Eight biological pathways were revealed to contain genes targeted by the miRNAs in all three factors, 38 pathways contained genes targeted by the miRNAs in two factors, and 55, 18 and two pathways were targeted by the miRNAs in a single factor, respectively (all $\mathrm{q}<0.05$ ). The pathways containing genes targeted by miRNAs in the largest factor shared a common theme of biological processes related to metabolism and inflammation. By contrast, the pathways containing genes targeted by miRNAs in the second largest factor were related to endocrine function and hormone activity. The present study focused on the pathways uniquely targeted
\end{abstract}

Correspondence to: Dr Elena Flowers, Department of Physiological Nursing, University of California San Francisco, 2 Koret Way, San Francisco, CA 94143-0610, USA

E-mail: elena.flowers@ucsf.edu

Abbreviations: BMI, body mass index; FBG, fasting blood glucose; GWAS, genome wide association studies; HbAlc, hemoglobin A1c; KEGG, Kyoto Encyclopedia of Genes and Genomes; miRNA/miR, microRNA; mRNA, messenger RNA; PRYSMS, Practicing Restorative Yoga versus Stretching for the Metabolic Syndrome; T2D, type 2 diabetes

Key words: miRNA, diabetes, FBG, biomarker, pathway analysis by each factor of miRNAs in order to identify unique mechanisms that may be associated with a subset of individuals. Further exploration of the genes and pathways related to these biological themes may provide insights about the subtypes of T2D and lead to the identification of novel therapeutic targets.

\section{Introduction}

Type 2 diabetes (T2D) affects $>30$ million individuals in the United States and its treatment costs $\$ 245$ billion annually. Individuals diagnosed with T2D experience numerous severe complications, such as cardiovascular diseases, retinopathy and renal disease (1). One of the primary challenges to prevent and treat T2D is its multifactorial etiology (2). Several genetic risk factors for T2D have been reported (3), including modifiable lifestyle and behavioral characteristics (4). Previous studies have identified various T2D subtypes with unique clinical characteristics (5-8). Although some differences exist in these subtypes among different racial and ethnic groups, the majority of characteristics are similar across groups, with the main difference being the prevalence of a subtype within each group (6,7). Ancestral genetic risk may be associated with the prevalence of these subtypes; however, the impact of environmental (e.g., lifestyle/behavioral) factors is well established. The increasing importance of the presence of T2D subtypes and the contribution of gene-environment interactions to T2D risk within the subtypes has led to interest in identifying and characterizing biomarkers that can reflect these complex relationships (9). Biomarkers that can characterize these relationships could present important implications for early risk detection before the onset of impaired fasting glucose (IFG) or T2D in order to prevent major complications and morbidities.

MicroRNAs (miRNAs/miRs) are short (18-26 nucleotides) regulatory elements that function during the translation of messenger RNAs (mRNAs) to amino acids. As miRNAs function by regulating gene expression, they regulate the underlying genetic risk factors for diseases as well as responses to the environment, including individual behaviors $(10,11)$. Circulating miRNAs found in the serum and plasma are easily 
measured in blood, and are potential risk biomarkers for the development of T2D $(12,13)$, thereby exhibiting changes in their expression levels before the onset of T2D $(14,15)$. Some circulating miRNAs may reflect the underlying physiological changes that are common across the subtypes of T2D, whereas others may provide information about the mechanisms that are unique to individual subtypes, based on both genetic predisposition and environmental factors.

miRNAs coregulate mRNAs using a combined approach that influences the sets of genes that operate in biological pathways (16). This indicates that coordinated changes in the expression of individual miRNAs would be associated with changes in their mRNAs and biological pathway targets to achieve a coherent impact on biological processes. A previous study on miRNAs related to T2D risk have mainly focused on single or a few candidate miRNAs (17). Furthermore, a previous study used an agnostic approach to identify individual miRNAs associated with IFG to define biomarkers that could identify the individuals at high risk of developing T2D (18); however, the study primarily focused on the associations between individual miRNAs and known clinical risk factors [e.g., fasting blood glucose (FBG) levels and hemoglobin A1c (HbA1c) levels], as well as the predicted mRNA targets located within pathways known to be associated with T2D risk (e.g., 'Type II diabetes mellitus' and 'Insulin signaling pathway').

The present study aimed to determine whether miRNAs can characterize individuals at risk for T2D into subgroups and whether any common mechanisms persist, inferred by genes and pathways targeted by miRNAs, both across and within the subgroups. The present study intended to build upon the results from previous studies by leveraging agnostic statistical approaches to determine the optimal sets of miRNAs based on their co-expression and to identify the common pathways that are responsible for T2D risk; in addition, the study aimed to elucidate the potential subsets of pathways that may be associated with the observed subtypes of T2D based on combined genetic and environmental risk factors. The overall goal of this research was early identification of T2D risk, as well as more refined detection of risk profiles, which could lead to the optimization of treatment strategies based on the improved understanding of underlying mechanisms within the subgroups.

\section{Materials and methods}

Study sample. The study sample included a subset of participants from the previously completed Practicing Restorative Yoga versus Stretching for the Metabolic Syndrome (PRYSMS) clinical trial (identifier NCT01024816; clinicaltrials.gov), which compared the effects of restorative yoga with those of active stretching on FBG in overweight adults at risk of T2D (19). Participants in the PRYSMS study were recruited from the San Francisco and San Diego areas, and met the International Diabetes Federation criteria for metabolic syndrome (20). The present study included a subset of participants with stored plasma specimens from the baseline visit with availability of at least two additional follow-up timepoints $(n=82)$. Exclusion criteria of the PRYSMS trial included FBG level $\geq 126 \mathrm{mg} / \mathrm{dl}$; HbAlc level $\geq 7.0 \%$; fasting triglyceride level $\geq 300 \mathrm{mg} / \mathrm{dl}$; weight $\geq 400 \mathrm{lb}$; neurological conditions that limited mobility; hospitalization for coronary heart disease within the past 6 months; current pregnancy or lactation; history of bariatric surgery; substance abuse; and use of medications affecting metabolic factors. Demographic and lifestyle characteristics, and medical history were collected by trained study personnel at the baseline visit.

Clinical data were collected at baseline, and at 3, 6, 9 and 12 months from the baseline. Participant weight and height were measured on a standard balance beam scale and stadiometer, respectively. Waist circumference was measured using a Gullick II tape spring-tension measure at the site of maximum circumference midway between the lower ribs and the anterior superior iliac spine. The mean of two waist circumference measurements was calculated. Body mass index (BMI) was calculated as weight in kilograms divided by height in meters squared.

FBG was measured using an automated analyzer with an immobilized enzyme biosensor (YSI 2300 STAT Plus; YSI Life Sciences). The levels of total cholesterol, triglycerides and high-density lipoprotein (HDL)-cholesterol were measured using enzymatic calorimetric methods (Quest Diagnostics), and low-density lipoprotein (LDL)-cholesterol levels were calculated using the Friedewald equation (21). All participants completed a 2-h oral glucose tolerance test. Blood used for plasma banking was collected by venipuncture into vacutainers containing the preservative EDTA, centrifuged at $4^{\circ} \mathrm{C}$ for $30,000 \mathrm{x} \mathrm{g}$ minutes to separate plasma from the cellular blood components, and was stored at $-80^{\circ} \mathrm{C}$.

Molecular data collection. The Firefly Bioworks Multiplex Circulating MicroRNA Assay (Abcam) was used for direct quantification of miRNAs from the plasma samples according to the manufacturer's protocol. After $25 \mu \mathrm{l}$ participant plasma was resuspended in $25 \mu 1$ hybridization buffer, miRNAs were hybridized to complementary oligonucleotides covalently attached to the encoded hydrogel microparticles. The bound target was ligated to the oligonucleotide adapter sequences that serve as universal PCR priming sites. Thereafter, reverse transcription PCR was performed using a fluorescent forward primer from the proprietary FirePlex miRNA Core Reagent Kit. The thermocycling conditions were as follows: One cycle at $93^{\circ} \mathrm{C}$ for $15 \mathrm{sec}$; followed by 32 cycles of $93^{\circ} \mathrm{C}$ for $5 \mathrm{sec}$, $57^{\circ} \mathrm{C}$ for $30 \mathrm{sec}$ and $68^{\circ} \mathrm{C}$ for $60 \mathrm{sec}$. Subsequently, 1 cycle at $68^{\circ} \mathrm{C}$ for $5 \mathrm{~min}$ and 1 cycle at $94^{\circ} \mathrm{C}$ for $4 \mathrm{~min}$ was performed, after which 1 cycle at $4^{\circ} \mathrm{C}$ was maintained until the assay was complete. Once amplified, the fluorescent target was rehybridized to the original capture particles and scanned on a Guava 6HT flow cytometer (Merck KGaA). Expression levels of 59 miRNAs were measured from the plasma specimens collected at each of the five time points from a subset of 86 participants of the PRYSMS trial. The selection of these 59 miRNAs was based on a previous discovery analysis from a broader set of 402 miRNAs from an independent subset of participants from the PRYSMS trial (13). The samples were not previously thawed. All miRNAs and sample wells included in the assay passed the quality control criteria.

Statistical analysis. The expression of individual miRNAs was normalized using a set of miRNA probes (i.e., hsamiR-92a-3p, hsa-mir-93-5p, hsa-miR-17-5p) identified 
by the geNorm algorithm for this experiment (22) using Fireplex Analysis Workbench software (https://www.abcam. com/kits/fireplex-analysis-workbench-software). All included miRNA targets passed the quality control check and were retained in the analysis.

Data were summarized using descriptive statistics (means and standard deviations for continuous variables, and counts and percentages for categorical variables). Missing values for FBG were imputed using the mean FBG level for an individual participant. An agnostic approach was applied to detect relationships between miRNAs based on observed expression levels in the present study compared with a priori hypotheses. A factor analysis was performed using a Varimax rotation on the covariance matrix to identify miRNAs that were related to baseline FBG level, final (12-month) FBG level and change in FBG levels. Eigenvalues $>1$ were the criteria for selecting important factors and miRNAs with values $>0.6$ were included in these factors.

Pathway analysis. An overrepresentation pathway analysis was performed for each factor of co-expressed miRNAs using the Kyoto Encyclopedia of Genes and Genomes (KEGG) database (June 2011 update) (23). Human miRNA data from three available regions (i.e., 5'untranslated, coding sequences and 3'untranslated) were downloaded from the miRWalk database (24) and merged into a single file. The inclusion criterion to identify mRNA targets was set to 1.0 for the binding probability score. The predicted mRNA targets were extracted for each miRNA included within the factors. Identified genes were then filtered to retain only one unique entry per targeted gene and Entrez IDs were determined using the Homo.sapiens v1.3.1 package (https://bioconductor.org/packages/Homo.sapiens/). These IDs were used to assess the overrepresentation within pathways using the ClusterProfiler package (version 3.16.1; https://bioconductor. org/packages/clusterProfiler/) and KEGG database. The default gene set background selected by ClusterProfiler was applied. Multiple comparisons were addressed by estimating the false discovery rate (25). The pathways within each factor were ranked by q-value. The Accelerating Medicines Partnership database was explored using the T2D phenotype to identify genes that revealed statistical associations with T2D in genome-wide association studies (GWAS) (26). These genes were cross-referenced with the genes present within the top $10 \mathrm{KEGG}$ pathways $(\mathrm{q}<0.05)$ identified as targets of the miRNAs within each factor.

In silico target gene identification. Using the miRWalk database (24), mRNAs were identified that are predicted targets of the miRNAs included in each factor. Thereafter, two lists (i.e., intersection of overlapping mRNAs and union of all mRNAs) of mRNA targets for each factor were generated. The Common Metabolic Diseases portal was used to cross-reference the mRNAs identified by the miRWalk database that were also associated with T2D in GWAS (26). In addition, a literature review was performed to assess the previously observed associations between identified mRNA targets for the miRNAs within the smallest identified factor and fourteen terms (i.e., endocrine, diabetes, glucose, glycemic impairment, fasting glucose, gestational diabetes, insulin, $\beta$-cells, pancreatic
Table I. Demographic information concerning patients $(n=82)$ and clinical results determined from samples.

\begin{tabular}{lc}
\hline Characteristic & Value \\
\hline Age, years & $55 \pm 7$ \\
Male sex & $27(22)$ \\
Completed college & $66(54)$ \\
Race & \\
Asian & $13(11)$ \\
Black & $5(4)$ \\
Latinx & $10(8)$ \\
Caucasian & $70(57)$ \\
Other/Mixed & $2(2)$ \\
Glucose (plasma), mg/dl & $104 \pm 13$ \\
Total cholesterol, mg/dl & $206 \pm 39$ \\
Triglycerides, mg/dl & $167 \pm 63$ \\
LDL-cholesterol, mg/dl & $125 \pm 35$ \\
HDL-cholesterol, mg/dl & $49 \pm 11$ \\
Waist circumference, cm & $110 \pm 13$ \\
Body mass index, kg/m ${ }^{2}$ & $35.1 \pm 7.2$ \\
Systolic blood pressure, $\mathrm{mmHg}$ & $124 \pm 15$ \\
Diastolic blood pressure, $\mathrm{mmHg}$ & $72 \pm 8$ \\
\hline
\end{tabular}

Data are presented as \% (n) or mean \pm standard deviation. HDL, highdensity lipoprotein; LDL, low-density lipoprotein.

$\beta$-cells, pancreas, overweight, obesity, adipose tissue, metabolic syndrome and heart disease) related to T2D risk.

\section{Results}

Demographic and clinical characteristics of the samples are summarized in Table I (13). The mean age of the patients was $55 \pm 7$ years and the majority of patients were women $(n=60$; $73 \%)$ who were Caucasian $(n=57 ; 70 \%)$. The patients presented with prediabetes and an average FBG level of $104 \pm 13 \mathrm{mg} / \mathrm{dl}$. The change in FBG level after 12 months was $-1 \pm 26 \mathrm{mg} / \mathrm{dl}$.

In total, 59 miRNAs were included in the assay; of these, 27 belonged to the identified factors (Fig. 1). The largest ('red'), second largest ('blue') and smallest ('green') factors comprised 17, seven and two miRNAs, respectively (Table II). The three factors with eigenvalues $>1$ explained $45 \%$ of the total variability in miRNA expression. The red, blue and green factors explained 30, 10 and 5\% of miRNA variability, respectively.

Pathway analysis of the genes targeted by the miRNA-based factors revealed that 99,59 and 17 KEGG pathways were targeted by the miRNAs in the red, blue and green factors, respectively $(\mathrm{q}<0.05$ for all; Table SI). When the KEGG pathways were ranked on the basis of q-values within each factor, eight pathways were targeted by the miRNAs in all three factors, and 38 were targeted by the miRNAs in two factors (Fig. 2). In total, 26 pathways were included by focusing on the top 10 most differentially perturbed pathways ranked by q-value in each factor (Fig. 3). The miRNAs in the red factor targeted seven unique pathways, including those with known relationships with the risk of T2D (e.g., 'AGE-RAGE signaling 


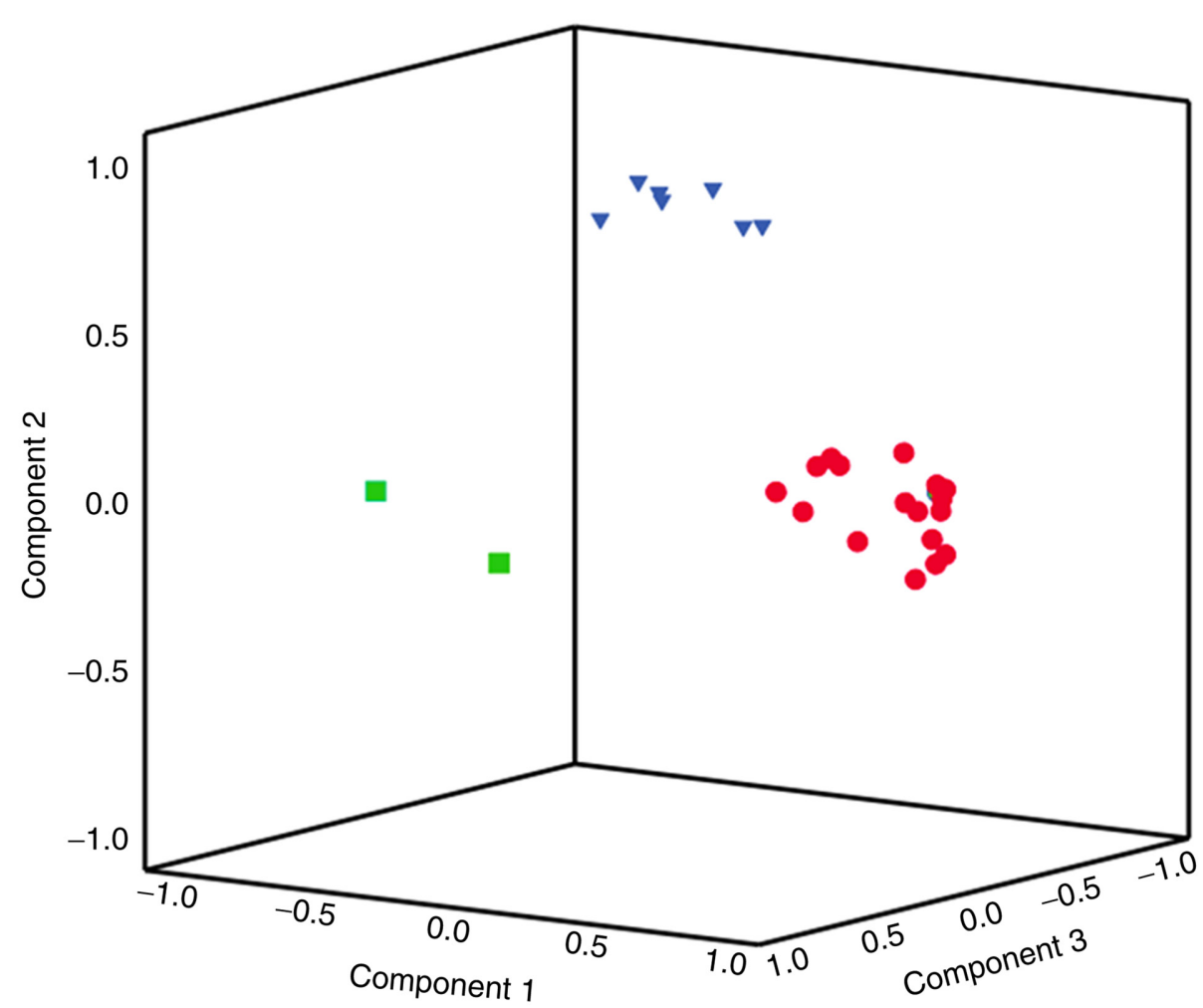

Figure 1. MicroRNA factors. Clusters of data points represent each of the three factors. Circles, red factor; triangles, blue factor; squares, green factor. The three components (i.e., 1, 2,3) are distinct latent variables that represent patterns of correlation in the data.

pathway' in diabetic complications, insulin resistance, glucagon signaling pathway, and lipid and atherosclerosis) (Table III). The miRNAs in the blue factor targeted two unique pathways that were related to endocrine and hormone activity: $\mathrm{GnRH}$ signaling and thyroid hormone synthesis (Table III).

Moreover, 17,958 total mRNA targets were identified for all miRNAs included in the three factors from the miRWalk tool. The 18, seven and two miRNAs from the red, blue, and green factors were predicted to target 17,728, 6,283 and 2,905 genes in total, respectively.

In total, 559 loci in 438 genes that are significantly associated with T2D in GWAS were identified from the Common Metabolic Diseases portal (26). These 438 genes were cross-referenced with those located in the KEGG pathways and comprised statistically significant gene targets of the miRNAs in each factor. The miRNAs in the red, blue and green factors targeted a total of $3,885,1,015$ and 252 genes in 99,59 and 17 pathways, respectively; of these, $113(2.9 \%), 52(5.1 \%)$ and 18 (7.1\%) from the red, blue and green factors were associated with T2D, respectively (Table SII). Within the top 10 targeted pathways ranked on the basis of q-value, the proportion of these genes in the red, blue and green factors increased to $4.9 \%$ (51 genes), $6.2 \%$ (23 genes) and $8.5 \%$ (15 genes), respectively.

A total of 192 genes were identified that represent the intersection of the predicted mRNA targets of the two miRNAs contained within the green factor (miR-16 and miR-342). A literature review on these predicted mRNAs along with 15 terms associated with T2D risk revealed that three, one, three, five and six genes were reported in association with eight, seven, six, five and four search terms related to T2D, respectively (Fig. 4). Other genes from this set of mRNA targets were associated with $<4$ terms. None of these 192 genes were located within the top 10 KEGG pathways targeted by miR-16 and miR-342.

\section{Discussion}

MicroRNAs coregulate their mRNA targets to perform coordinated regulation of biological processes (16); therefore, it was hypothesized that miRNAs with overlapping functions could be grouped based on associated expression levels. The present study used an agnostic, data-driven statistical approach to identify the co-expression patterns of circulating miRNAs, which resulted in the identification of three distinct factors. Furthermore, an in silico approach was used to identify the individual genes and biological pathways that were targeted by the miRNAs within each factor, and assessed them for overlap, common themes and concordance with the results from previous GWAS.

While pathway annotations provide a framework to infer the involvement of physiological processes, their interpretations are challenging. For example, the pathways in the KEGG database are assigned names based on their initial characterization (e.g., a cancer model); however, some pathways may serve a crucial role in the pathogenesis of diseases and conditions other than the one for which they were initially named, and these misnomers can obfuscate biologically related processes when hypothesis-driven approaches are applied. Considering the variable etiology of T2D, agnostic approaches to identify the underlying mechanisms may provide novel insights into the interactions between multiple risk factors and disease development. 
Table II. miRNAs comprising each factor.

\begin{tabular}{lccc}
\hline & \multicolumn{3}{c}{ Factor loading values } \\
\cline { 2 - 4 } miRNA & Red & Blue & Green \\
\hline let-7f-5p & & 0.897 & \\
miR-126-3p & 0.749 & & \\
miR-126-5p & & 0.797 & \\
miR-130b-3p & 0.881 & & \\
miR-151a-3p & 0.938 & & \\
miR-151a-5p & 0.957 & & \\
miR-151b & 0.919 & & \\
miR-186-5p & & 0.878 & \\
miR-197-3p & 0.895 & & \\
miR-221-3p & 0.950 & & \\
miR-23a-3p & 0.774 & & \\
miR-24-3p & 0.977 & & \\
miR-27a-3p & 0.946 & & \\
miR-29b-3p & & 0.828 & \\
miR-320c & 0.640 & & \\
miR-323a-3p & 0.610 & & \\
miR-326 & 0.953 & & \\
miR-330-3p & 0.737 & & \\
miR-342-3p & & & \\
miR-374b-5p & & 0.788 \\
miR-379-5p & & & \\
miR-424-5p & & 0.892 & \\
miR-425-3p & 0.838 & & \\
miR-652-3p & 0.914 & & \\
miR-98-5p & & & \\
miR-15b-5p & 0.866 & & \\
miR-16-5p & & & \\
\hline
\end{tabular}

Factor loading values were determined by factor analysis using a Varimax rotation. miR/miRNA, microRNA.

In the present study, the red factor could be renamed as the metabolism and inflammation factor, as it targeted pathways related to insulin and glucose metabolism, and inflammation/immune function. Some pathways, such as the AGE-RAGE signaling pathway in diabetic complications, insulin resistance, glucagon signaling pathway, and lipid and atherosclerosis, are linked to T2D. The functions of the other pathways targeted by the miRNAs in the red factor may plausibly be involved in the etiology of T2D, despite the names lacking a clear association with T2D. Notably, the endocytosis pathway has been reported to contribute to autoimmune activity (23), and the human T-cell leukemia virus 1 infection pathway may contribute to immune activity as well as inflammation (23). Furthermore, the adrenergic signaling in cardiomyocytes pathway has been shown to serve a pivotal role in stimulating the sympathetic nervous system, thereby causing increased inflammation (23). Both immune function and inflammation are related to insulin sensitivity and other processes that are responsible for T2D risk (27).
The blue factor could be renamed as the endocrine-hormone factor, as it comprised miRNAs that uniquely target two KEGG pathways associated with endocrine function. The GnRH signaling pathway represents the release of sex hormones from the hypothalamus to the pituitary gland.(23) A previous animal model study identified seven genes located in the 'Type II diabetes' KEGG pathway that were downregulated in association with high GnRH release, which suggests a plausible role for this pathway in the development of T2D (28). The thyroid hormone synthesis pathway produces triiodothyronine and thyroxine, which are essential for metabolic homeostasis (23). Thyroid disease is more prevalent in individuals with T2D compared with in individuals with normal glucose tolerance, and thyroid hormones help to regulate glucose absorption, insulin secretion and glycogen metabolism (29). Collectively, the pathways targeted by the endocrine factor may contribute to the overall pathophysiology of T2D.

As the green factor did not uniquely target any pathways, an alternative name was not suggested. A total of 192 genes were identified that were targeted by one or both of the miRNAs in the green factor (i.e., miR-16 and miR-342); furthermore, several of these 192 genes have previously been associated with T2D and related conditions. The associations between the most commonly identified genes and the search terms can be categorized into discrete aspects of T2D risk. Three genes were primarily associated with terms related to glucose and diabetes. These included adenylate cyclase 5 (ADCY5), which has been reported to be associated with T2D and fasting blood glucose in GWAS (30), and nicotinamide phosphoribosyltransferase, which has been shown to be independently associated with T2D after controlling for obesity and insulin sensitivity, and may be more related to glucose control (31). Polymorphisms in the potassium voltage-gated channel subfamily B member 1 may be associated with insulin resistance (32), the protein coded by tea shirt zinc finger homeobox 1 regulates $\beta$-cell maturation (33), and alleles in JAZ zinc finger 1 reveal differential transcription specifically in the pancreatic tissue (34). Clearly linked to lipid metabolism, numerous polymorphisms in LDL receptor protein 5 are associated with obesity and body composition $(35,36)$. Moreover, an overlap has been observed between these categories (i.e., glucose control, insulin sensitivity and obesity), and several of these genes presumably exhibit coordinated functions involving multiple mechanisms. For example, $A D C Y 5$ is associated with T2D, as well as with BMI and obesity $(37,38)$. Some of the genes associated with numerous search terms may appear frequently, as they are related to fundamental cellular processes as opposed to mechanisms unique to T2D risk. Two examples include mitogen-activated protein kinase 9, which is involved in numerous cellular processes (e.g., proliferation, differentiation, transcription regulation) and cell division cycle 4 , which regulates signaling pathways to control multiple cellular functions (e.g., morphology, migration, endocytosis, cell cycle progression) (39).

Some pathways targeted by miRNAs in multiple factors may serve physiological roles that are common across multiple subtypes of T2D. The longevity regulating pathway and longevity regulating pathway-multiple species were targeted by the miRNAs in all three factors and two factors, respectively. Aging is a major risk factor for $\beta$-cell dysfunction and 


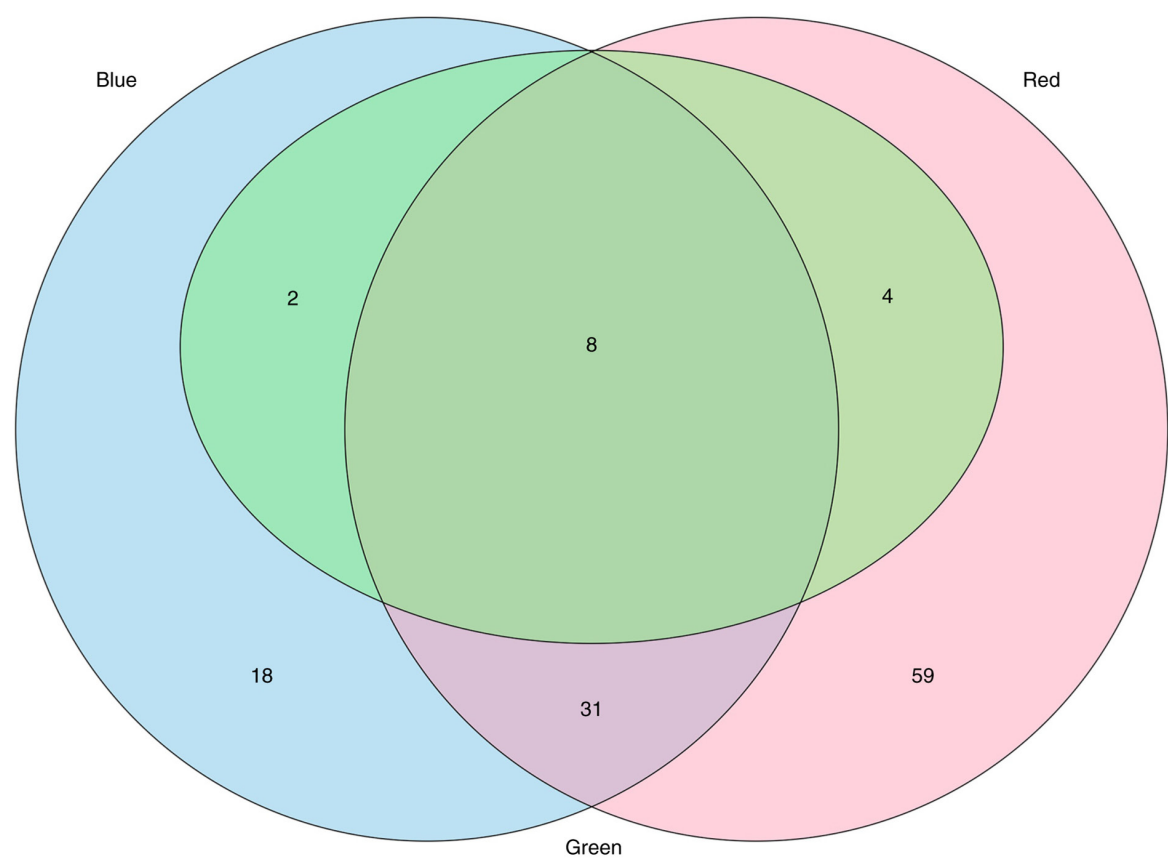

Figure 2. Venn diagram showing KEGG pathways by miRNAs. The color of the circle represents the corresponding miRNA factor. The number within each section is the number of KEGG pathways targeted by miRNAs within each factor or a combination of factors at q<0.05. KEGG, Kyoto Encyclopedia of Genes and Genomes; miRNA, microRNA.

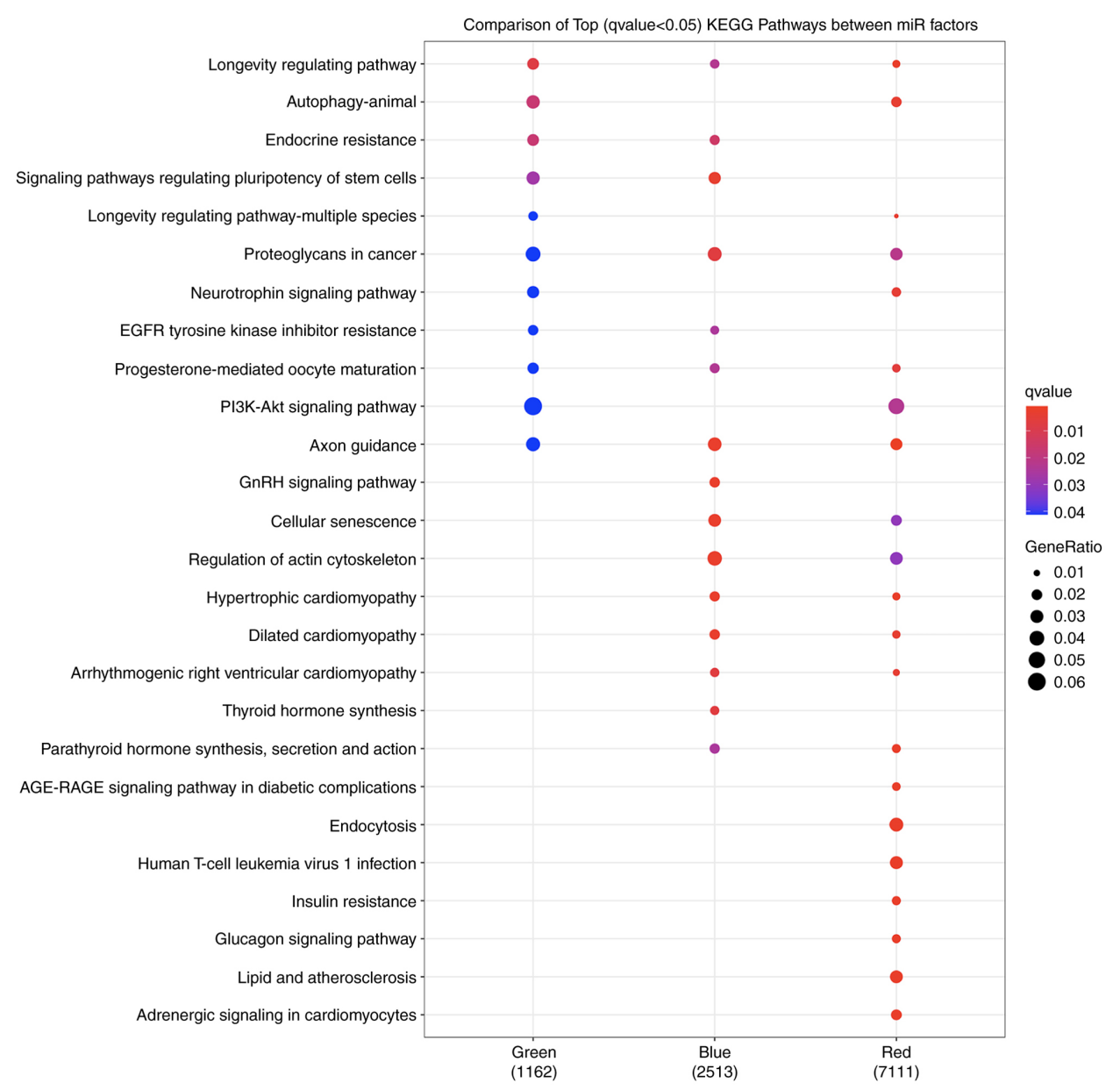

Figure 3. Ranked KEGG pathways by clusters of miRNAs. Up to 10 pathways that were significant $(q<0.05)$ targets of the miRNAs contained within a single factor are shown. Pathways targeted by more than one factor are signified by dots that occur on the same horizontal line. The size of the dot represents the number of genes within the pathway that are predicted targets of the miRNAs within a given factor relative to the total number of genes included in the analyses. The total number of gene targets for each factor is shown in parentheses on the X-axis. The color of the dot shows the magnitude of the false discovery rate-adjusted P-value. KEGG, Kyoto Encyclopedia of Genes and Genomes; miRNA/miR, microRNA. 
Table III. Kyoto Encyclopedia of Genes and Genomes pathway by factor.

\begin{tabular}{lc}
\hline Pathway & Percentage $^{\mathrm{b}}$ \\
\hline Metabolism and inflammation (red) & \\
AGE-RAGE signaling pathway in diabetic & 8.1 \\
complications & \\
Endocytosis & 4.2 \\
Human T-cell leukemia virus 1 infection & 12.4 \\
Insulin resistance & 6.6 \\
Glucagon signaling pathway & 7.6 \\
Lipid and atherosclerosis & 3.9 \\
Adrenergic signaling in cardiomyocytes & 6.9 \\
Endocrine-hormone (blue) & \\
GnRH signaling pathway & 4.2 \\
Thyroid hormone synthesis & 2.6 \\
\hline
\end{tabular}

apathways included reflect only the pathways that were uniquely targeted by the microRNAs within just one factor; ${ }^{\text {bpercentages of }}$ genes within a given pathway that have known associations with type 2 diabetes from genome-wide association studies.

T2D (40); notably, caloric restriction is one of the only known interventions to increase lifespan (41). Animal model studies have provided evidence that caloric restriction improves $\beta$-cell function and decreases insulin resistance $(42,43)$. These longevity pathways are involved in the responses triggered by caloric restriction, including improved cellular fitness and inflammation suppression (23). Autophagy is an important aspect used to protect $\beta$-cells from oxidative stress, and the resulting dysfunction associated with insulin resistance and progression to T2D (44); moreover, the autophagy-animal pathway was targeted by the miRNAs within two factors in the present study. Further studies are required to determine whether perturbation of this pathway is a precursor to, or consequence of $\mathrm{T} 2 \mathrm{D}$, as autophagy is also related to adipocyte function and obesity-related inflammation that can lead to T2D (44). Autophagy is strongly related to aging, and further research is required to determine whether any interactions exist between the autophagy and longevity pathways, and T2D risk. The mechanisms represented by these pathways are not unique to a single aspect of T2D risk, but may be broadly fundamental to maintaining healthy homeostasis overall.

Based on the pathway analysis, the present study cross-referenced whether genes present within the pathways targeted by the combination of miRNAs in each factor had been identified by GWAS focused on T2D. A limitation of GWAS findings is that complex physiological processes that require coordinated activity of multiple gene products are not necessarily characterized; however, genetic polymorphisms that result in functional changes have the potential to alter these physiological processes, and therefore parallels between genetic associations with T2D and transcriptomic and biological pathway associations with T2D may be identified. The present study identified numerous genes within the targeted pathways that have also been discovered in GWAS focused on T2D, providing more evidence for a potential putative role of these genes in T2D risk. In addition, this layered approach provides evidence to prioritize findings from GWAS for functional validation studies.

Several risk factors for T2D are important for the etiology of other complex diseases (e.g., coronary heart disease, stroke). For example, the atherogenic dyslipdemia profile, which is characterized by low HDL and elevated triglycerides, is indicative of insulin resistance that is associated risk for T2D (45). This dyslipdemia risk profile is also associated with increased risk for both coronary heart disease and stroke (45). Hypertension, one of the most significant risk factors for coronary artery disease and stroke, also frequently co-occurs with T2D (45). Potential clinical applications of miRNAs as biomarkers extend beyond T2D (46), and the findings from this study may have implications for these related conditions. Additional studies focusing on these outcomes in addition to T2D may elucidate potential mechanisms that result from the overlap in risk factors, with the potential to differentiate similarities and differences in the underlying risk profiles and potential new therapeutic targets (47).

The present study identified three factors of miRNAs, defined by their associated expression levels, with common themes within their biological pathways and predicted mRNA targets. Some of these targets are known to be associated with the pathophysiology of T2D, whereas others may reveal new mechanisms that underlie the complex interactions among T2D risk factors. Subsets of these miRNA factors, and their targeted genes and pathways, may be discretely associated with recently described subtypes of T2D. A potential limitation of the present study was the focus on the KEGG database for pathway annotation, in particular high-quality annotations (i.e., those with the most rigorous level of evidence). Additional pathway annotation databases are available, and future studies may consider overlapping evidence from multiple databases. Functional validation of the observed relationships is also required to determine causal relationships between miRNAs and their targets. We previously performed a discovery analysis using an independent sample of participants from the PRYSMS trial that measured the subset of reliably detectable circulating miRNAs, thus revealing the selection of the 59 miRNAs included in the design of the custom assay used in the present study (13). Additional miRNAs not included in this assay may be relevant for T2D risk. Future studies that are designed to include a larger number of miRNAs may identify additional miRNAs that are relevant to the three factors identified in the present study or that define an additional novel factor(s). Additionally, a possible bias exists in the findings from the literature review because genes with a known association with T2D risk are more likely to be studied, whereas genes with little known information about their function may be less commonly reported. In addition to replication and functional analyses, another important future direction is to identify the subgroups of individuals with similar patterns of expression of miRNAs and the miRNA-derived factors to determine any associations with the observed subgroups of T2D that present specific clinical characteristics (5-8). The long-term implications include improved understanding of the specific mechanisms that underlie T2D risk within subtypes based on their overall risk profile and interactions between the risk factors. 


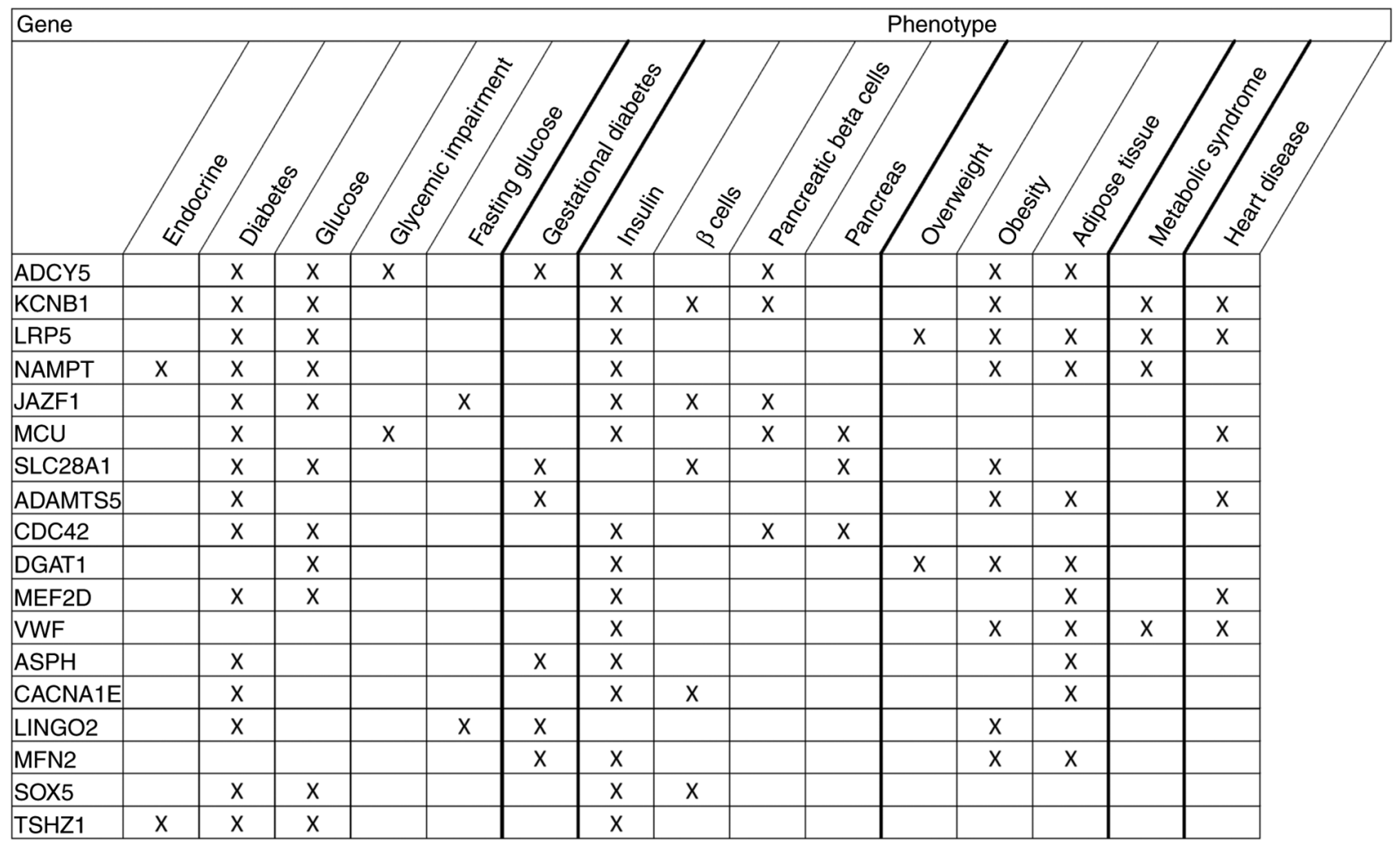

Figure 4. Predicted gene targets associated with diabetes and related conditions. Columns show search terms. Rows show gene names.

\section{Acknowledgements}

The authors would like to thank Ms. Julinna Cheung (University of California, USA) and Ms. Lena Noya (Brown University, USA) for their contributions to the literature review of the current study. The authors would also like to thank Ms. Lisa Chang (Keck Graduate Institute, USA) for assisting with the development of figures. Finally, the authors acknowledge Ms. Xingyue Gong (University of California, USA) for contributing to the literature review and data analysis of the present study.

\section{Funding}

Dr Flowers was supported by the National Center for Advancing Translational Sciences of the National Institutes of Health (grant no. KL2TR000143) and the Hellman Family Foundation. The PRYSMS study was supported by the National Center for Complementary and Alternative Medicine of the National Institutes of Health (grant no. R01AT004569). Molecular data collection from the PRYSMS study was supported by the National Institute of Diabetes, Digestive, and Kidney Disease of the National Institutes of Health (grant no. R21DK117346). Dr Kanaya is supported by National Heart Lung, and Blood Institute of the National Institutes of Health (grant no. 2K24HL112827).

\section{Availability of data and materials}

The datasets used and/or analyzed during the current study are available from the corresponding author on reasonable request.

\section{Authors' contributions}

EF was the principal investigator of the grant that supported this project, and conceived of and executed the overall study and design, oversaw all molecular data collection, directed the analysis plan, and wrote and revised the manuscript. KA contributed to the analysis plan and performed bioinformatics analyses. IEA contributed to the analysis plan, performed all statistical modeling, contributed to the interpretation of the results and approved the final manuscript. AMK was the principal investigator of the PRYSMS trial and oversaw all clinical data collection, contributed to the design and interpretation of results for this study, and approved the final manuscript. BEA provided overall scientific guidance for this study, consulted on the bioinformatic analyses, contributed to interpretation of the results and approved the final manuscript. EF, IEA and AMK confirm the authenticity of the raw data. All authors read and approved the final manuscript.

\section{Ethics approval and consent to participate}

All participants in the parent PRYSMS trial provided written informed consent for all trial procedures, including banking of biospecimens for future analyses. The present study was approved by the Institutional Review Board at the University of California San Francisco (approval no. \#10-02436).

\section{Patient consent for publication}

Not applicable. 


\section{Competing interests}

The authors declare that they have no competing interests.

\section{References}

1. National Diabetes Statistics Report: Centers for Diseaes Control and Prevention, U.S. Department of Health and Human Services, Atlanta, GA, 2017.

2. Manolio TA, Collins FS, Cox NJ, Goldstein DB, Hindorff LA, Hunter DJ, McCarthy MI, Ramos EM, Cardon LR, Chakravarti A, et al: Finding the missing heritability of complex diseases. Nature 461: 747-753, 2009.

3. Xue A, Wu Y, Zhu Z, Zhang F, Kemper KE, Zheng Z, Yengo L, Lloyd-Jones LR, Sidorenko J, Wu Y, et al: Genome-wide association analyses identify 143 risk variants and putative regulatory mechanisms for type 2 diabetes. Nat Commun 9 : 2941, 2018

4. Bagchi D: Nutritional and therapeutic interventions for diabetes and metabolic syndrome. Elsevier, Waltham, MA, 2018.

5. Ahlqvist E, Storm P, Käräjämäki A, Martinell M, Dorkhan M, Carlsson A, Vikman P, Prasad RB, Aly DM, Almgren P, et al: Novel subgroups of adult-onset diabetes and their association with outcomes: A data-driven cluster analysis of six variables. Lancet Diabetes Endocrinol 6: 361-369, 2018.

6. Zou X, Zhou X, Zhu Z and Ji L: Novel subgroups of patients with adult-onset diabetes in Chinese and US populations. Lancet Diabetes Endocrinol 7: 9-11, 2019.

7. Anjana RM, Baskar V, Nair ATN, Jebarani S, Siddiqui MK Pradeepa R, Unnikrishnan R, Palmer C, Pearson E and Mohan V: Novel subgroups of type 2 diabetes and their association with microvascular outcomes in an Asian Indian population: A data-driven cluster analysis: The INSPIRED study. BMJ Open Diabetes Res Care 8: e001506, 2020.

8. Herder C, Maalmi H, Strassburger K, Zaharia OP, Ratter JM, Karusheva Y, Elhadad MA, Bódis K, Bongaerts BWC, Rathmann W, et al: Differences in biomarkers of inflammation between novel subgroups of recent-onset diabetes. Diabetes 70: 1198-1208, 2021

9. Hill-Briggs F, Adler NE, BerkowitzSA, Chin MH, Gary-Webb TL, Navas-Acien A, Thornton PL and Haire-Joshu D: Social determinants of health and diabetes: A scientific review. Diabetes Care 44: 258-279, 2020

10. Flowers E, Won GY and Fukuoka Y: MicroRNAs associated with exercise and diet: A systematic review. Physiol Genomics 47: 1-11, 2015.

11. Parr EB, Camera DM, Burke LM, Phillips SM, Coffey VG and Hawley JA: Circulating microRNA responses between 'high' and 'low' responders to a 16-Wk diet and exercise weight loss intervention. PLoS One 11: e0152545, 2016.

12. Vaishya S, Sarwade RD and Seshadri V: MicroRNA, proteins, and metabolites as novel biomarkers for prediabetes, diabetes, and related complications. Front Endocrinol (Lausanne) 9: 180, 2018.

13. Flowers E, Allen IE, Kanaya AM and Aouizerat BE: Circulating microRNAs predict glycemic improvement and response to a behavioral intervention. Biomark Res 9: 65 2021.

14. Flowers E, Aouizerat BE, Abbasi F, Lamendola C, Grove KM, Fukuoka Y and Reaven GM: Circulating microRNA-320a and microRNA-486 predict thiazolidinedione response: Moving towards precision health for diabetes prevention. Metabolism 64 : 1051-1059, 2015

15. Flowers E, Gadgil M, Aouizerat BE and Kanaya AM: Circulating micrornas associated with glycemic impairment and progression in Asian Indians. Biomark Res 3: 1-8, 2015.

16. Wang Y, Luo J, Zhang $\mathrm{H}$ and Lu J: MicroRNAs in the same clusters evolve to coordinately regulate functionally related genes. Mol Biol Evol 33: 2232-2247, 2016.

17. Zhu $\mathrm{H}$ and Leung SW: Identification of microRNA biomarkers in type 2 diabetes: A meta-analysis of controlled profiling studies. Diabetologia 58: 900-911, 2015.

18. Mononen N, Lyytikäinen LP, Seppälä I, Mishra PP, Juonala M, Waldenberger M, Klopp N, Illig T, Leiviskä J, Loo BM, et al: Whole blood microRNA levels associate with glycemic status and correlate with target mRNAs in pathways important to type 2 diabetes. Sci Rep 9: 8887, 2019.
19. Kanaya AM, Araneta MR, Pawlowsky SB, Barrett-Connor E, Grady D, VittinghoffE,Schembri M, Chang A, Carrion-Petersen ML, Coggins T, et al: Restorative yoga and metabolic risk factors: the practicing restorative yoga vs. stretching for the metabolic syndrome (PRYSMS) randomized trial.J Diabetes Complications 28: 406-412, 2014.

20. Alberti KG, Eckel RH, Grundy SM, Zimmet PZ, Cleeman JI, Donato KA, Fruchart JC, James WP, Loria CM, Smith SC Jr, et al: Harmonizing the metabolic syndrome: A joint interim statement of the international diabetes federation task force on epidemiology and prevention; national heart, lung, and blood institute; American heart association; world heart federation; international atherosclerosis society; and international association for the study of obesity. Circulation 120: 1640-1645, 2009.

21. Friedewald WT, Levy RI and Fredrickson DS: Estimation of the concentration of low-density lipoprotein cholesterol in plasma, without use of the preparative ultracentrifuge. Clin Chem 18: 499-502, 1972.

22. Vandesompele J, De Preter K, Pattyn F, Poppe B, Van Roy N, De Paepe A and Speleman F: Accurate normalization of real-time quantitative RT-PCR data by geometric averaging of multiple internal control genes. Genome Biol 3: RESEARCH0034, 2002.

23. Kanehisa M and Goto S: KEGG: Kyoto encyclopedia of genes and genomes. Nucleic Acids Res 28: 27-30, 2000.

24. Dweep H, Sticht C, Pandey P and Gretz N: MiRWalk-database: Prediction of possible miRNA binding sites by 'walking' the genes of three genomes. J Biomed Inform 44: 839-847, 2011.

25. Benjamini Y and Hochberg Y: Controlling the false discovery rate: A practical and powerful approach to multiple testing. J Royal Statistical Society. Series B (Methodological) 57: 289-300, 1995

26. Common Metabolic Diseases Knowledge Portal.https://hugeamp. org/phenotype.html?phenotype=T2D. Accessed May 26, 2021.

27. Sell H, Habich $\mathrm{C}$ and Eckel J: Adaptive immunity in obesity and insulin resistance. Nat Rev Endocrinol 8: 709-716, 2012

28. Xiang W, Zhang B, Lv F, Feng G, Chen L, Yang F, Zhang K, Cao $\mathrm{C}$, Wang $\mathrm{P}$ and $\mathrm{Chu} \mathrm{M}$ : The potential regulatory mechanisms of the gonadotropin-releasing hormone in gonadotropin transcriptions identified with bioinformatics analyses. Reprod Biol Endocrinol 15: 46, 2017.

29. Johnson JL: Diabetes control in thyroid disease. Diabetes Spectrum 19: 148-153, 2006.

30. Dupuis J, Langenberg C, Prokopenko I, Saxena R, Soranzo N, Jackson AU, Wheeler E, Glazer NL, Bouatia-Naji N, Gloyn AL, et al: New genetic loci implicated in fasting glucose homeostasis and their impact on type 2 diabetes risk. Nat Genet 42: 105-116, 2010.

31. Esteghamati A, Alamdari A, Zandieh A, Elahi S, Khalilzadeh O, Nakhjavani $M$ and Meysamie A: Serum visfatin is associated with type 2 diabetes mellitus independent of insulin resistance and obesity. Diabetes Res Clin Pract 91: 154-158, 2011.

32. Yu Y, Wang J, Kang R, Dong J, Zhang Y, Liu F, Yan Y, Zhu R, Xia L, Peng X, et al: Association of KCNB1 polymorphisms with lipid metabolisms and insulin resistance: A case-control design of population-based cross-sectional study in Chinese Han population. Lipids Health Dis 14: 112, 2015.

33. Raum JC, Soleimanpour SA, Groff DN, Coré N, Fasano L, Garratt AN, Dai C, Powers AC and Stoffers DA: Tshzl regulates pancreatic $\beta$-cell maturation. Diabetes 64: 2905-2914, 2015.

34. Fogarty MP, Panhuis TM, Vadlamudi S, Buchkovich ML and Mohlke KL: Allele-specific transcriptional activity at type 2 diabetes-associated single nucleotide polymorphisms in regions of pancreatic islet open chromatin at the JAZF1 locus. Diabetes 62: 1756-1762, 2013.

35. Adabi E, Omidfar A, Farahani NA, Faghihi F, Asghar Malek Hosseini SA, Maghbooli Z and Shirvani A: The association of LRP5 (rs556442) polymorphism with body composition and obesity in postmenopausal women. Diabetes Metab Syndr 13: 2381-2385, 2019.

36. Zhang L, Wang J, Zhang M, Wang G, Shen Y, Wu D, Wang C, Li L, Ren Y, Wang B, et al: Association of type 2 diabetes mellitus with the interaction between low-density lipoprotein receptor-related protein 5 (LRP5) polymorphisms and overweight and obesity in rural Chinese adults. J Diabetes 9: 994-1002, 2017.

37. Perry JR, Voight BF, Yengo L, Amin N, Dupuis J, Ganser M, Grallert H, Navarro P, Li M, Qi L, et al: Stratifying type 2 diabetes cases by BMI identifies genetic risk variants in LAMA1 and enrichment for risk variants in lean compared to obese cases. PLoS Genet 8: e1002741, 2012. 
38. Manning AK, Hivert MF, Scott RA, Grimsby JL, Bouatia-Naji N, Chen H, Rybin D, Liu CT, Bielak LF, Prokopenko I, et al: A genome-wide approach accounting for body mass index identifies genetic variants influencing fasting glycemic traits and insulin resistance. Nat Genet 44: 659-669, 2012

39. Gene (Internet): National Library of Medicine (US). National Center for Biotechnology Information, Bethesda, MD, 1988.

40. Chang AM and Halter JB: Aging and insulin secretion. Am J Physiol Endocrinol Metab 284: E7-E12, 2003.

41. Newgard CB and Pessin JE: Recent progress in metabolic signaling pathways regulating aging and life span. J Gerontol A Biol Sci Med Sci 69 (Suppl 1): S21-S27, 2014.

42. Corezola do Amaral ME, Kravets V, Dwulet JM, Farnsworth NL, Piscopio R, Schleicher WE, Miranda JG and Benninger RK: Caloric restriction recovers impaired $\beta$-cell- $\beta$-cell gap junction coupling, calcium oscillation coordination, and insulin secretion in prediabetic mice. Am J Physiol Endocrinol Metab 319: E709-E720, 2020.

43. He XY, Zhao XL, Gu Q, Shen JP, Hu Y and Hu RM: Calorie restriction from a young age preserves the functions of pancreatic $\beta$ cells in aging rats. Tohoku J Exp Med 227: 245-252, 2012.
44. Stienstra R, Haim Y, Riahi Y, Netea M, Rudich A and Leibowitz G: Autophagy in adipose tissue and the beta cell: Implications for obesity and diabetes. Diabetologia 57: 1505-1516, 2014.

45. American Diabetes Association. 10. Cardiovascular disease and risk management: Standards of medical care in diabetes-2021. Diabetes Care 44 (Suppl 1): S125-S150, 2021.

46. Condrat CE, Thompson DC, Barbu MG, Bugnar OL, Boboc A, Cretoiu D, Suciu N, Cretoiu SM and Voinea SC: miRNAs as biomarkers in disease: Latest findings regarding their role in diagnosis and prognosis. Cells 9: 276, 2020.

47. Rupaimoole R and Slack FJ: MicroRNA therapeutics: Towards a new era for the management of cancer and other diseases. Nat Rev Drug Discov 16: 203-222, 2017.

This work is licensed under a Creative Commons Attribution-NonCommercial-NoDerivatives 4.0 International (CC BY-NC-ND 4.0) License. 